\title{
The body's life support system under attack
}

Keywords: liver, drug use, obesity, diabetes, hepatitis

\section{Opinion}

The dramatic increase in media attention highlights the severity of the opioid epidemic, the rising rates of hepatitis, related liver cancer, diabetes, fatty liver, and prescription drug and alcohol misuse and abuse. However, even with the national attention being drawn to these devastating diseases, one important message continues to be overlooked. That message, is that all of these diseases, are liver related and preventable.

Individuals have the power to prevent the occurrence of these diseases by managing their health care and developing healthier food and lifestyle behaviours. How can they be motivated to do so, without providing them with a basic understanding about the important role the liver, the organ under attack, plays in maintaining their health and life itself? This non complaining complex organ and its miraculous hard working liver cells convert everything they eat, breathe and absorb through their skin into hundreds of life sustaining body functions 24/7. They need to understand how their food and lifestyle choices can lead to irreparable liver cell damage, cirrhosis and eventually liver cancer herein lies the problem. Unless we motivate individuals to protect this remarkable organ that is under attack by educating children in schools, government employees and the military, these chronic illnesses will continue to impact the health and wellbeing of millions, shorten their lives while dramatically increasing the burden on our healthcare system. Individuals and community leaders express public outrage and pleas for "quick fixes" to respond to the rapidly escalating opioid and other drug abuse epidemics, hepatitis, and obesity. Current efforts focus on dealing with these conditions after the fact with emergency responses to those who have overdosed, who are addicted, hepatitis infected or obese with barely a mention of promoting primary preventative liver information initiatives.

A cancer preventing vaccine against hepatitis B is available but is underutilized because many individuals are unaware of the serious consequences related to this infectious disease. There is no vaccine to prevent Hepatitis $\mathrm{C}$ and effective treatments are extremely costly and unavailable to many patients. Injection drug use and misuse of needles and other invasive instruments are reported to be the major source of HCV infections according to the Center for Disease Control and Prevention. Efforts to elimination both hepatitis B and $\mathrm{C}$ depend primarily on bringing the source of the majority of these infections . . . drug abuse under control. Drug use, obesity, diabetes and atherosclerosis depend on each individual making informed and healthier selection of food and drinks that protect their miraculous life sustaining liver. Tragically, primary prevention that includes liver information is being ignored ... lives will continue to be lost that could
Volume 3 Issue 6 - 2017

Thelma King Thiel

Liver Wellness Professional Consultant, Orange Memorial

Hospital School of Nursing, USA

Correspondence: Thelma King Thiel, Liver Wellness Professional Consultant, Liver Health Initiative, Orange Memorial Hospital School of Nursing, USA, Email livrlady@gmail.com

Received: October 26, 2017 | Published: November 22, 2017

have been prevented and healthcare cost will increase exponentially. Primary prevention is the key to saving lives and healthcare dollars.

\section{Liver health initiative mission statement}

The Liver Health Initiative is a 501c3 organization dedicated to promoting healthy food and lifestyle behaviors and prevention of liver related diseases through multifaceted liver health education programs. The mission of the Liver Health Initiative is to make liver health education a priority on the national agenda. Promoting education about the liver to empower individuals to make informed and healthier food and lifestyle choices, can improve compliance and treatment outcomes, and reduce the incidence of preventable chronic liver related diseases including obesity, fatty liver, hepatitis, atherosclerosis, early onset diabetes, high cholesterol, and cardiovascular diseases.

\section{Biography}

Thelma King Thiel, RN, BA has dedicated her life to promoting liver health for the past 47 years since the loss of her infant son Dean to biliary atresia. Ms. Thiel has served as the CEO of both the American Liver Foundation and the Hepatitis Foundation International for a total of 35years, and has trained thousands of healthcare providers about liver health. Ms. Thiel recently came out of retirement to organize the Liver Health Initiative, to fill the enormous liver health knowledge gap and empower individuals to understand why and how to protect their miraculous life sustaining liver. Promoting primary prevention through liver health education is Ms. Thiel's ultimate goal.

\section{Acknowledgements}

None.

\section{Conflict of interest}

Author declares that there is no conflict of interest. 